\title{
Signal Transduction Events in Health and Disease
}

\begin{abstract}
Overproduction of nitric oxide causes oxidative stress in joint tissues. Under this stress, synovial cells are more prone to pro-inflammatory cytokine stimulation, which could result in the development of Rheumatoid Arthritis. This laboratory nitric oxide in cells. They have begun testing different concentrations of a cyclic peptides (derived from the shared epitope sequence) in order to check if cyclic peptides maintain the same ability to triger nitric oxide signaling as the linear counterpart; cyclic peptides have longer half-lives than linear peptides. For detecting nitric oxide response in cells, DAF-2 DA dye is loaded into M1 cells a $37^{\circ} \mathrm{C}$ for $1 \mathrm{hour}$; then cells are treated with different concentrations of cyclic peptides. Linear peptides are used as positive control, and no peptide treatment the negative control. Nitric oxide levels are continually monitored by a fluorescence micro-plate reader during an eight hour time period. The results obtained from this experiment will be used to design a cyclic peptide to stimulate the signaling cascade that leads to the increase of nitric oxide in cells. Eventuallys these data will help us to further understand the genetic causes of Rheumatoid Arthritis.
\end{abstract}

\section{Introduction}

Shared epitope positive individuals are more prone to rheumatoid arthritis, and other autoimmune diseases. The linear peptide $65-79 * 0401$ has been shown to increase nitric oxide production in cells. High levels of nitric oxide in cells cause cell death, and in rheumatoid arthritis patients can cause extreme discomfort due to the swelling of joints. The aim of this project is to compare the relative NO production rate between the linear, and cyclic peptide. The results of this experiment will be used to further understand the signaling cascade that occurs in hared epitope positive individuals that leads to the development of theumatoid arthritis.

\section{Methods}

NO Assay:

$3 \times 10^{4}$ human fibroblasts were loaded in each well of 96 -well plates one day before experiment in order to allow the cells to attach to the surface. The supernant was removed, and cells were washed with Phenol-red free DMEM buffer. Cells were first loaded with $20 \mu \mathrm{M}$ of the fluorescent NO probe 4,5-diaminofluorescein diacetate (DAF-2DA), and then incubated in the dark at $37^{\circ} \mathrm{C}$ for 1 hour. After dye loading the supernant was removed, and the cells were once again washed. Each well in the 96- well plate received $50 \mu \mathrm{l}$ of Phenol-red free medium, and $50 \mu \mathrm{l}$ of either more medium to act as control wells, the linear peptide, or the cyclic peptide in varying concentrations $(.1,1,10,100,1000 \mu \mathrm{M})$. NO signal was recorded by using a Fusion aHT system at an excitation wavelength of $488 \mathrm{~nm}$ and emission wavelength of $515 \mathrm{~nm}$.

ROS Assay:

ROS production was quantified similarly, with the exception that cells were loaded

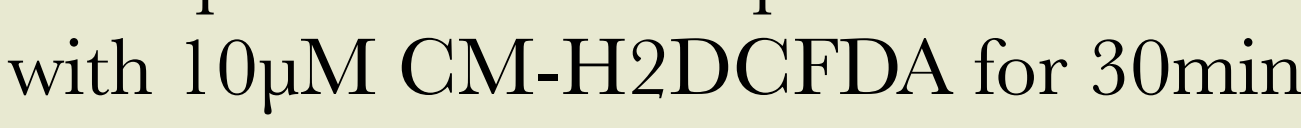

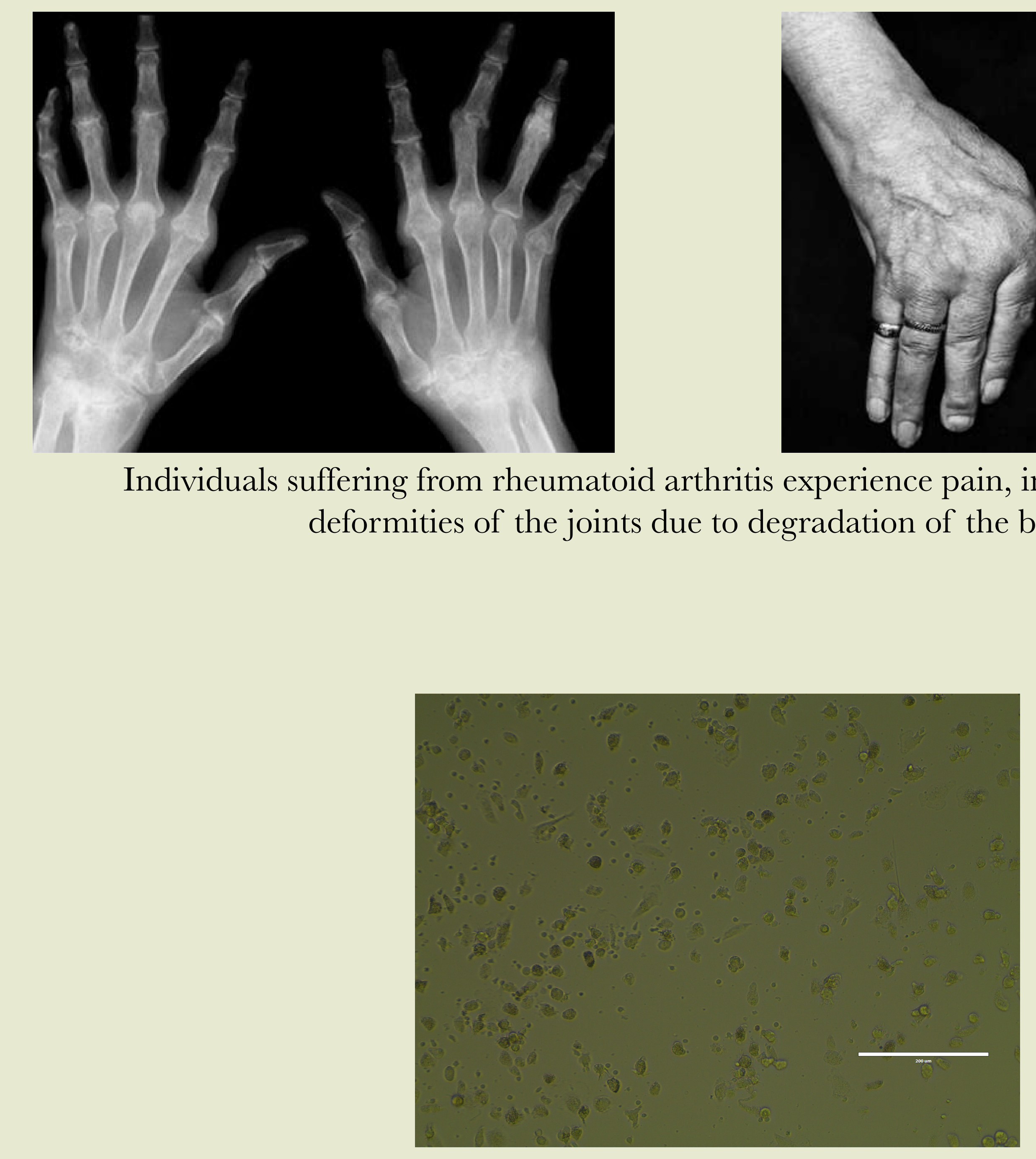

Immature Human Dendritic Cells
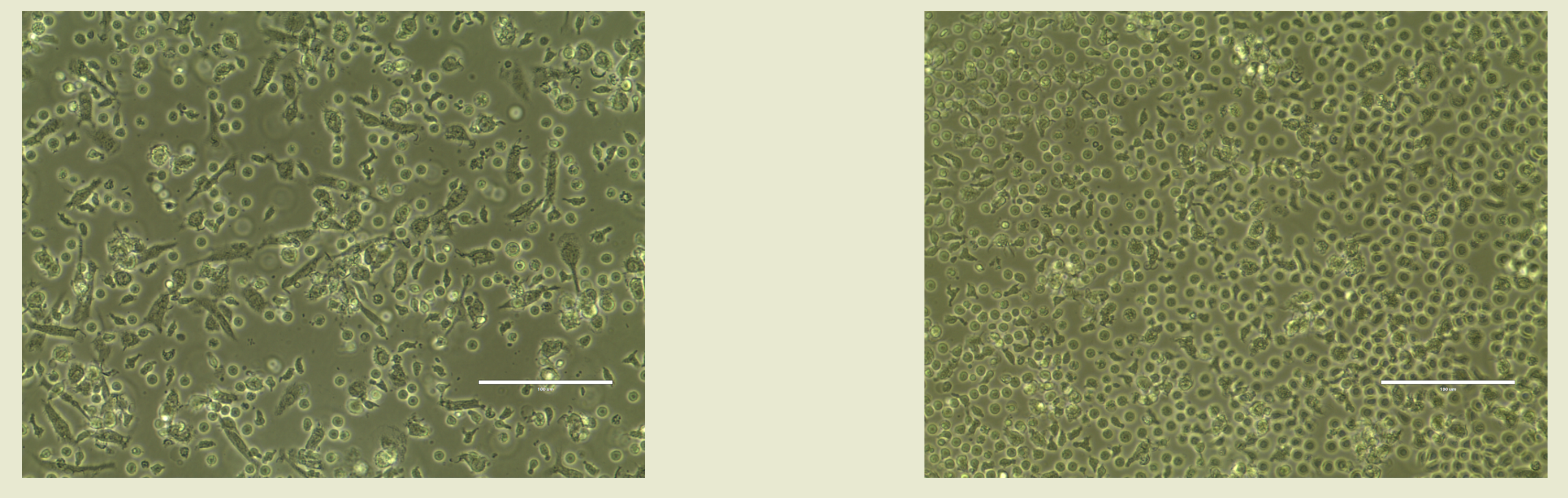

Human PBMC depleted of CD4+ and highly adherent cells after 3 days of culture. The picture on the left is of cells treated with $50 \mathrm{\mu g} / \mathrm{ml}$ DRB $1-0401$ on day 0 . The picture on

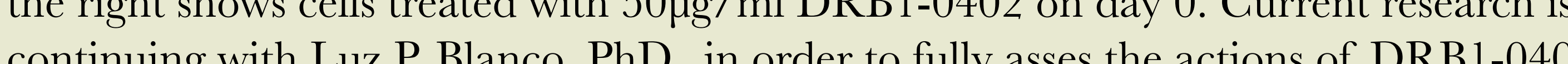
and DRB1-0402 in te

\section{Results}

Analysis of the resulting NO, and ROS Assay showed that cells treated with $57 \mathrm{mM}$ 65-79*0401, and the cyclic peptide had higher relative NO production rates compared to he con rol cells. The highest $N$ production

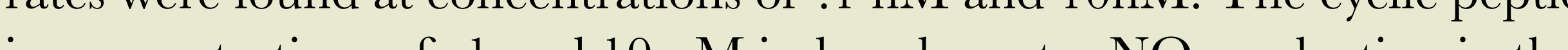
M1 cell the 1 an 57 mM 65-79*0401 peptide. with concentrations of 1,100, and $1000 \mathrm{nM}$ had relative NO production rates lower than those of cells treated with $57 \mathrm{mM}$ 65-79*0401.

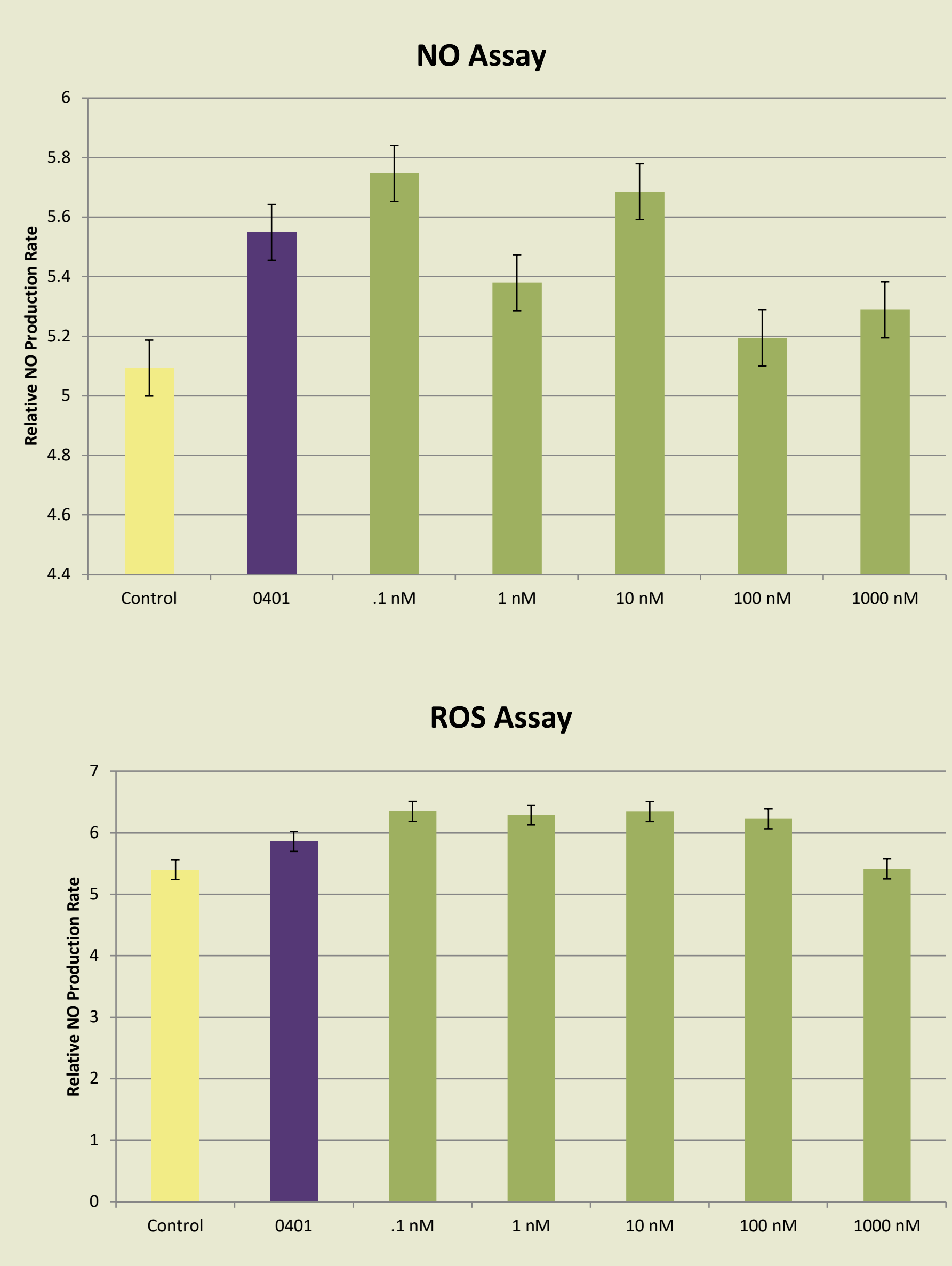

\section{Conclusion}

The data collected from NO and ROS assays supports our hypothesis that peptide $65-79 * 0401$ induces nitric oxide production in human fibroblasts cells. The data also shows that the cyclic peptide is fully capable of producing relative $\mathrm{NO}$ production in rates exceeding those of the linear peptide $65-79 * 0401.10 \mathrm{nM}$ of the cyclic peptide has a more potent ability to stimulate NO signaling than the $57 \mathrm{mM}$ linear peptide, which indicates that the cyclic peptide is 5000 folds more potent than the cyclic peptide. It should be added that the cyclic peptide has a more stale structure, and longer half-life. The knowledge gained from these experiments is currently being used in studies with human dendritic cells to fully understand the signaling cascade that occurs in shared epitope positive individuals. 